\title{
Amiodarone pulmonary toxicity: functional and ultrastructural evaluation
}

\author{
FELIX LOK-WAN LIU, RICHARD D COHEN, EUGENE DOWNAR, \\ JAGDISH W BUTANY, JEFFREY D EDELSON, ANTHONY S REBUCK
}

From Toronto Western and Toronto General Hospitals, Toronto, Ontario, Canada

ABSTRACT Pulmonary function, chest radiographic appearances, and the cellular composition of bronchoalveolar lavage fluid were assessed in 13 patients who were receiving amiodarone treatment. Eight of the patients had developed clinical and radiological evidence of lung disease and five were symptom free. The proportions of lymphocytes (mean 8.6 (SD 6.9)) and neutrophils (mean 3.4 (3.3)) obtained by bronchoalveolar lavage were similar in patients with and without lung complications. Electron microscopic examination of alveolar macrophages showed intralysosomal inclusion bodies in all subjects, regardless of clinical state. There was no significant difference in the mean number of inclusion bodies per macrophage transection between those with and those without lung disease. The differential cell count in bronchoalveolar lavage fluid and the presence of macrophage inclusion bodies were therefore not useful as markers of disease activity. Among those who developed clinical and radiological evidence of lung disease, the cumulative drug dose per kilogram of body weight and the duration of treatment (mean 16.5 (SD 9.0) months) were significantly correlated with the degree of lung restriction as measured by total lung capacity and forced vital capacity. It is concluded that, while the severity of the restrictive pulmonary defect that is induced by amiodarone is largely dose related, the development of lung toxicity is to some extent idiosyncratic.

Since its discovery in 1961, amiodarone hydrochloride, an iodinated derivative of benzofuran, has found increasing use in the treatment of life threatening ventricular and supraventricular arrhythmias refractory to other agents. ${ }^{12}$ In high dosage ranges several serious side effects occur, including corneal microdeposits, changes in thyroid function, blue skin pigmentation, and photosensitivity, ${ }^{34}$ associated with intralysosomal inclusion bodies. Recently a syndrome of rapidly progressive pneumonitis has been described. ${ }^{5-9}$

In many interstitial lung diseases, a process of alveolar inflammation can be identified before the development of structural lung damage. ${ }^{10}$ Open lung biopsy performed in several patients with amiodarone induced lung disease has shown evidence of such alveolar and interstitial inflammation. ${ }^{7-9}$ The availability of bronchoalveolar lavage allows sampling of immune effector cells of the lower respiratory tract. ${ }^{11}$

Address for reprint requests: Dr AS Rebuck, Division of Respiratory Medicine, Toronto Western Hospital, 399 Bathurst Street, Toronto, Ontario M5T 2S8, Canada.

Accepted 2 September 1985
We performed bronchoalveolar lavage in 13 patients who had been taking amiodarone for various periods of time, eight of whom had developed pulmonary infiltrates for which no explanation other than a drug reaction was apparent. We hoped to identify the previously reported alveolitis in those patients who had developed a radiological infiltrate, and to determine whether symptom free patients taking amiodarone might have subclinical alveolitis.

\section{Methods}

Thirteen patients ( 12 men and one woman, mean age 58.9 (SD 13.6) years) who had taken amiodarone hydrochloride for at least seven months (mean 20.5 (13.9) months) for control of cardiac arrhythmia were referred for assessment.Eight of the patients had developed clinical and radiological evidence of an otherwise unexplained lung disease; the remaining five had no evidence of any lung disease. This group was studied because of concern on the part of their referring doctors about a clinically silent alveolitis that might precede overt amiodarone induced lung disease. All patients signed informed consent before 
Table 1 Results for 13 patients (Nos 1-8 developed lung disease)

\begin{tabular}{|c|c|c|c|c|c|c|c|c|c|c|c|c|}
\hline \multirow[t]{2}{*}{$\begin{array}{l}\text { Case } \\
\text { No }\end{array}$} & \multirow[t]{2}{*}{$\begin{array}{l}\text { Age } \\
(y)\end{array}$} & \multicolumn{3}{|c|}{ Amiodarone treatment } & \multicolumn{3}{|c|}{$\begin{array}{l}\text { Pulmonary function } \\
(\% \text { predicted normal })\end{array}$} & \multicolumn{2}{|c|}{$\begin{array}{l}\text { Arterial blood gas } \\
\text { tensions } \\
(\mathrm{mm} \mathrm{Hg}(\mathrm{kPa}))\end{array}$} & \multicolumn{3}{|l|}{ Lavage fluid } \\
\hline & & $\begin{array}{l}\text { Average daily } \\
\text { dose }(\mathrm{g})\end{array}$ & $\begin{array}{l}\text { Cumulative } \\
\text { dose }(g)\end{array}$ & $\begin{array}{l}\text { Duration } \\
(m)\end{array}$ & $T L C$ & $F V C$ & $T L C O$ & $\mathrm{PaO}_{2}$ & $\mathrm{PaCO}_{2}$ & $\begin{array}{l}\text { Macrophages } \\
(\%)\end{array}$ & $\begin{array}{l}\text { Lymphocytes } \\
(\%)\end{array}$ & $\begin{array}{l}\text { Neutrophils } \\
(\%)\end{array}$ \\
\hline $\begin{array}{c}1 \\
2 \\
3 \\
4^{*} \\
5 \\
6 \\
7 \\
8 \\
9 \\
10 \\
11 \\
12 \\
13\end{array}$ & $\begin{array}{l}45 \\
65 \\
30 \\
75 \\
76 \\
60 \\
57 \\
56 \\
55 \\
77 \\
46 \\
68 \\
56\end{array}$ & $\begin{array}{l}0.567 \\
0.406 \\
0.286 \\
0.303 \\
0.353 \\
0.471 \\
0.486 \\
0.424 \\
0.365 \\
0.417 \\
0.423 \\
0.428 \\
0.338\end{array}$ & $\begin{array}{l}136 \\
195 \\
256 \\
182 \\
286 \\
113 \\
102 \\
191 \\
493 \\
400 \\
482 \\
164 \\
71\end{array}$ & $\begin{array}{r}8 \\
17 \\
32 \\
20 \\
27 \\
8 \\
7 \\
15 \\
45 \\
33 \\
38 \\
12.5 \\
7\end{array}$ & $\begin{array}{r}95 \\
47 \\
83 \\
63 \\
102 \\
115 \\
107 \\
117 \\
112 \\
75 \\
116 \\
115\end{array}$ & $\begin{array}{r}87 \\
100 \\
32 \\
59 \\
71 \\
112 \\
133 \\
111 \\
108 \\
137 \\
85 \\
105 \\
105\end{array}$ & $\begin{array}{r}82 \\
42 \\
80 \\
122 \\
74 \\
48 \\
99 \\
108 \\
92 \\
102 \\
87 \\
78 \\
96\end{array}$ & $\begin{array}{l}84(11.2) \\
64(8.5) \\
64(8.5) \\
91(12) \\
76(10) \\
71(9.5) \\
- \\
\overline{70}(9.3) \\
82(10.9) \\
98(13) \\
73(9.7) \\
-\end{array}$ & $\begin{array}{l}38(5.1) \\
37(4.9) \\
51(6.8) \\
38(5.1) \\
39(5.2) \\
31(4.1) \\
= \\
\overline{37}(4.9) \\
33(4.4) \\
35(4.7) \\
35(4.7)\end{array}$ & $\begin{array}{l}96 \\
76 \\
71 \\
94 \\
87 \\
92 \\
96 \\
88 \\
93 \\
98 \\
97 \\
83 \\
80\end{array}$ & $\begin{array}{r}1 \\
15 \\
4 \\
1 \\
3 \\
1 \\
1 \\
7 \\
2 \\
1 \\
1 \\
3 \\
3\end{array}$ & $\begin{array}{r}4 \\
9 \\
25 \\
5 \\
10 \\
7 \\
3 \\
5 \\
5 \\
1 \\
3 \\
14 \\
17\end{array}$ \\
\hline
\end{tabular}

* Female.

TLC - total lung capacity; FVC - forced vital capacity; TLco-transfer factor (diffusing capacity); $\mathrm{PaO}_{2}-$ arterial oxygen tension; $\mathrm{PaCO}_{2}-\mathrm{arterial}_{\text {carbon }}$ dioxide tension.

bronchoscopy. Each patient underwent chest radiographic examination, pulmonary function testing, flexible fibreoptic bronchoscopy, and bronchoalveolar lavage.

Fibreoptic bronchoscopy was performed after topical airway anaesthesia had been induced. After inspection of the upper airway and tracheobronchial tree, the tip of the bronchoscope was wedged in a segmental bronchus of the lingula. The segment was lavaged with five $20 \mathrm{ml}$ aliquots of $0.9 \%$ saline solution as previously described. ${ }^{11}$ The $50-70 \mathrm{ml}$ of lavage fluid recovered was divided into two aliquots, and submitted for light and transmission electron microscopy.

For light microscopic examination, the fluid was filtered under $24 \mathrm{~mm} \mathrm{Hg}$ negative pressure, $5 \mu \mathrm{m}$ Millipore filters (two slides) and $5 \mu \mathrm{m}$ Gelman filters (two slides) being used. After immediate fixation in $95 \%$ ethanol, the slides were stained by the Papanicolaou method. If the slides showed good alveolar sampling a differential cell count was carried out with 300-500 non-epithelial cells. The total cell content of the bronchoalveolar lavage fluid was not estimated.

Samples for electron microscopy were centrifuged at $400 \mathrm{~g}$ for 10 minutes. After the supernatant had been discarded, the pellet was resuspended in $2 \%$ glutaraldehyde $(0.1 \mathrm{~mol} / 1$ phosphate buffered) and fixed for two hours. The specimen was then rinsed in $0.1 \mathrm{~mol} / 1$ phosphate buffer and postfixed in $1 \%$ buffered osmium tetroxide for one hour. After a rinse in distilled water, the specimens were passed through graded ethanol, ending with propylene oxide, and subsequently embedded in Epon. Ultrathin sections were cut with a diamond knife mounted on copper grids, and the sections stained with lead citrate and uranyl acetate. These sections were examined with a Phillips 300 transmission electron microscope. Mac- rophages were morphologically easily distinguishable from lymphocytes. The average number of intralysosomal inclusion bodies per alveolar macrophage transection was determined by counting the total number of granular and lamellar electron dense and electron lucent bodies in 10-15 alveolar macrophages. Care was taken to select maximal cross sections and to avoid cell sections that were blatantly fragments or pseudopodia. The pathologist who interpreted the electron microscopic sections was unaware of the patient's clinical state.

Blood samples for measurement of serum amiodarone concentration were drawn within one week of the bronchoalveolar lavage. The amiodarone in serum was measured by high pressure liquid chromatography as previously described. ${ }^{12}$

The clinical history and chest radiographs of each subject were reviewed by a panel of three independent pulmonary physicians. Each subject was allocated to one of two categories: either "unaffected," if there was no clinical or radiological evidence of lung disease, or "pneumonitis," if there was evidence of an infiltrate on the chest radiograph for which no other cause was evident.

Data for the two patient groups are expressed as means with one standard deviation in parentheses. Mean values of groups were compared by an unpaired $t$ test (two tailed). Relationships among variables were sought with the method of least squares linear regression analysis.

\section{Results}

Eight subjects were assigned to the "pneumonitis" group based on a history of dyspnoea, cough and radiological infiltrate for which no other cause was evident. Five subjects who had no clinical or radio- 
Table 2 Amiodarone dosage and serum amiodarone concentrations

\begin{tabular}{llll}
\hline & Mean $(\mathrm{SD})$ & & \\
\cline { 2 - 4 } & All subjects & Pneumonitis $(\boldsymbol{n}=8)$ & Unaffected $(n=5)$ \\
\hline Age $(\mathrm{y})$ & $58.9(13.6)$ & $58.0(15.2)$ & $60.4(12.1)$ \\
Average daily dose $(\mathrm{mg})$ & $405(77)$ & $412(95)$ & $394(40)$ \\
Average daily dose/kg body weight $(\mathrm{mg})$ & $5.66(1.63)$ & $5.95(1.97)$ & $5.21(0.87)$ \\
Cumulative dose $(\mathrm{g})$ & $236(142)$ & $184(67)$ & $320(192)$ \\
Cumulative dose/kg body weight $(\mathrm{mg})$ & $3.22(1.79)$ & $2.44(1.03)$ & $4.48(2.13)$ \\
Duration of treatment $(\mathrm{m})$ & $20.5(12.9)$ & $16.5(9.0)$ & $26.8(16.5)$ \\
Serum amiodarone concentration $(\mathrm{mg} / \mathrm{l})$ & $2.03(1.26)$ & $2.10(1.49)$ & $1.86(1.12)$ \\
\hline
\end{tabular}

logical evidence of pulmonary disease were assigned to the "unaffected" category.

\section{CHEST RADIOGRAPHIC APPEARANCES}

The eight subjects in the pneumonitis group had recently developed abnormalities on their chest radiographs at the time of presentation. Five had interstitial infiltration with a lower lung field distribution, one had interstitial and alveolar infiltrates at the apices, and two had diffuse interstitial infiltrates and patchy parenchymal consolidation. Gallium 67 lung scans were performed in four subjects in the "pneumonitis" group, yielding positive results in two. The chest radiograph was normal in the remaining five subjects.

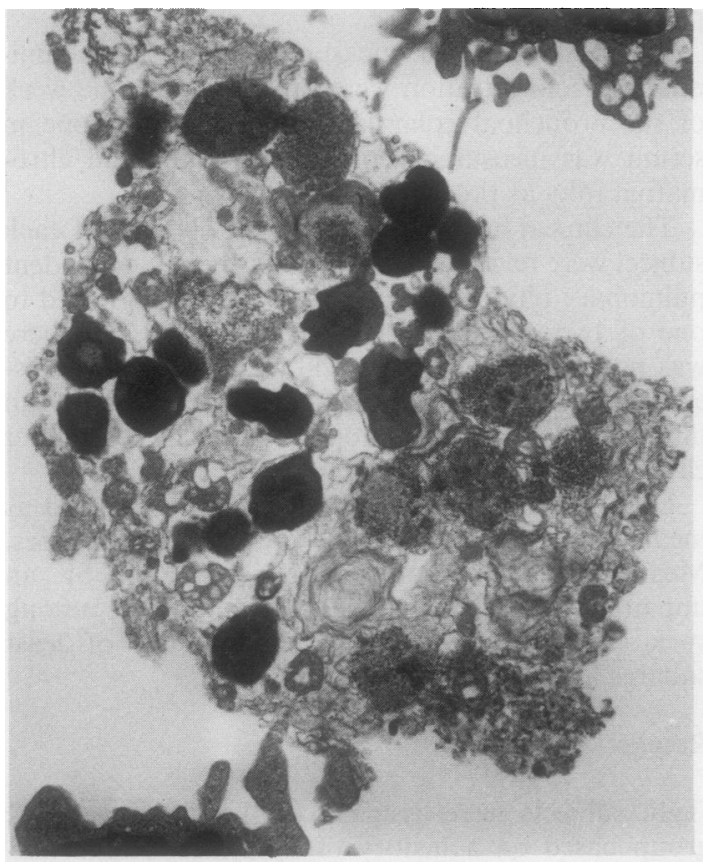

Fig 1 Electron micrograph of lavaged alveolar macrophage showing inclusion bodies.
DRUG DOSAGE AND TREATMENT DURATION

These results are shown in tables 1 and 2 . The age, average daily dose of amiodarone and average daily dose per kilogram of body weight did not differ between the pneumonitis and the unaffected group. The mean duration of treatment and cumulative drug dose were lower in the pneumonitis group. These differences did not reach statistical significance. The mean cumulative dose of drug per kilogram body weight was significantly lower in the pneumonitis group (2.44 (1.03) g) than in the unaffected patients $(4.48(2.13) \mathrm{g} ; \mathrm{p}<0.03)$. The mean serum amiodarone concentration was $2.10(1.26) \mathrm{mg} / \mathrm{l}$ in the pneumonitis group and $1.86(1.12) \mathrm{mg} / \mathrm{l}$ among unaffected subjects.

\section{PULMONARY FUNCTION TESTS AND ARTERIAL} BLOOD GAS TENSIONS

The mean total lung capacity (TLC) was $87.4 \%$ $(24.7 \%)$ of the predicted value in the pneumonitis group and $107.0 \%(18.0 \%)$ predicted in the unaffected subjects. The mean forced vital capacity (FVC) was $88.1 \%$ (32.8\%) predicted in patients with pneumonitis and $108.0 \%(18.6 \%)$ predicted in the unaffected patients. Among the pneumonitis patients the mean single breath diffusing capacity for carbon monoxide was $81.9 \%(27.7 \%)$ predicted compared with $91.0 \%(9.1 \%)$ predicted in the unaffected patients. Mean arterial oxygen and carbon dioxide tensions were not significantly different in the two groups.

BRONCHOALVEOLAR LAVAGE FLUID

The percentage of bronchoalveolar lavage fluid lymphocytes (mean 8.6 (SD 6.9)) and of neutrophils (3.4 (3.3)) in the pneumonitis group did not differ significantly from those of the unaffected subjects (lymphocytes 8.1 (7.0); neutrophils $1.9(1.0)$ ), the balance of cells in each being alveolar macrophages. Electron microscopic evaluation of alveolar macrophages obtained by bronchoalveolar lavage showed lamellar, electron lucent, electron dense, or mixed types of membrane bound inclusion bodies inside lysosomes (fig 1). Inclusion bodies were found in all subjects regardless of clinical state. The mean number (1)

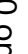

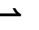
을

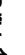
$\overrightarrow{0}$

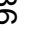




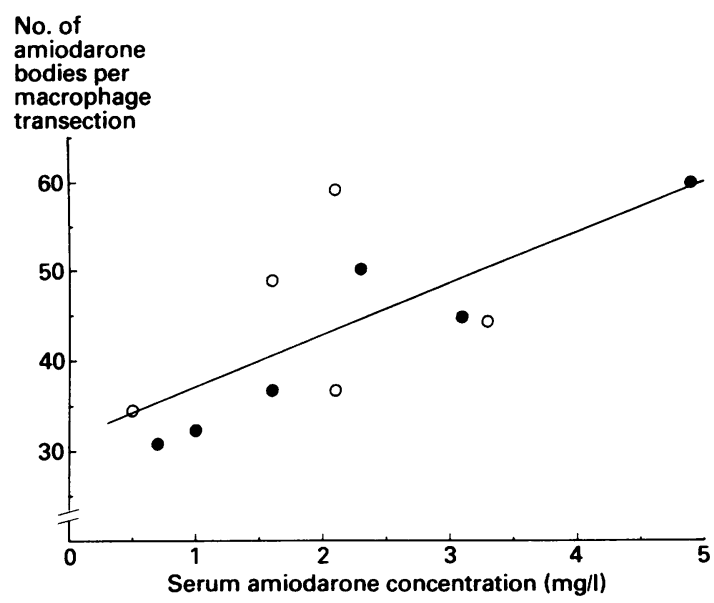

Fig 2 The mean number of amiodarone bodies per lavaged alveolar macrophage related to serum amiodarone concentration. There is a significant correlation $(r=0.72$, $p<0.03$ ). Open circles represent subjects without lung disease and closed circles those who developed lung disease ( $n=11$; in two patients electron microscopic evaluation was technically unsatisfactory).

of electron microscopic inclusion bodies per alveolar macrophage transection in the pneumonitis group (40.3 (11.9)) was not significantly different from the mean for the unaffected group (44.7 (10.0)). The mean number of electron microscopic inclusion bodies per alveolar macrophage transection was, however, correlated with the average daily dose $(r=0.65, p<$ 0.03 ) and with the serum amiodarone concentration

FVC

(\% pred)

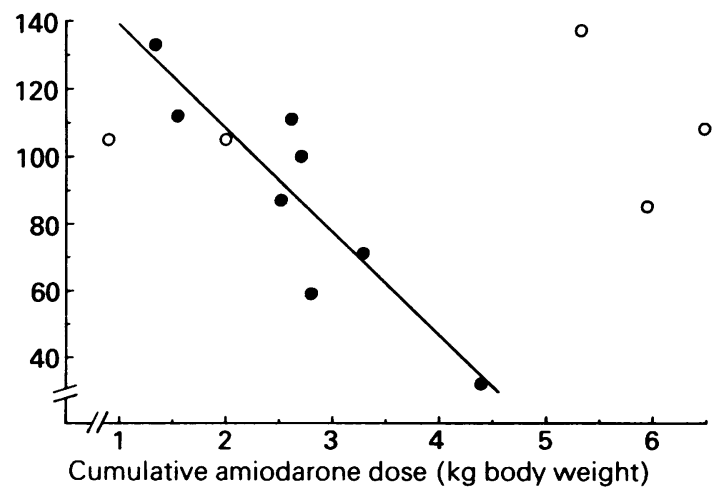

Fig 3 Forced vital capacity (FVC) of eight subjects who developed lung disease (closed circles), expressed as percentage of predicted normal, and cumulative amiodarone dose per kilogram of body weight $(r=0.90, p<0.01)$. Five subjects without lung disease (open circles) are shown for comparison and are not included in the correlation. $(r=0.72, p<0.03)$ when all patients were considered together (fig 2).

Six months after discontinuation of amiodarone treatment repeat bronchoalveolar lavage in one subject showed no change in the number of inclusion bodies per macrophage transection, despite a reduction in the serum amiodarone concentration to undetectable values.

There was no correlation between indices of pulmonary function (total lung capacity (TLC), FVC, $\mathrm{FEV}_{1}, \mathrm{FEV}_{1} / \mathrm{FVC}$ ) and the percentage of lavage fluid lymphocytes, percentage of lavage fluid neutrophils, serum amiodarone concentration, average daily dose, cumulative drug dose, duration of drug treatment, or the number of inclusion bodies per macrophage. Among the pneumonitis group, however, cumulative drug dose per kilogram of body weight (mean 2.44 $(1.03) \mathrm{g}$ ) and duration of drug treatment (mean 16.5 (9.0) months) were significantly related to the degree of lung restriction as measured by TLC $(r=-0.91$, $\mathrm{p}<0.01$; and $\mathrm{r}=-0.96, \mathrm{p}<0.001$ respectively) and by FVC $(r=-0.90, p<0.01$; and $r=-0.85$, $p<$ 0.01 respectively) (fig 3 ).

\section{SUBSEQUENT COURSE OF AMIODARONE}

RELATED LUNG DISEASE

Chest radiographic abnormalities and symptoms resolved in subjects 2 and 4 after amiodarone had been discontinued and prednisone treatment instituted. Subject 3 died of an unrelated cause two weeks after the drug had been discontinued. Subjects 1, 6, 7, and 8 showed complete resolution of radiographic abnormalities and symptoms after the dose had been decreased from an average of $4 \mathrm{~g}$ to $2 \mathrm{~g}$ per week. In subject 5 symptoms and radiographic abnormalities progressed slowly despite a decrease in drug dosage from $2.8 \mathrm{~g}$ to $2.0 \mathrm{~g}$ per week.

\section{Discussion}

Since the initial report suggesting an association between administration of amiodarone and adverse pulmonary consequences, at least $\mathbf{4 0}$ such cases have been reported. The syndrome ranges from a reversible pulmonary infiltrate on the chest radiograph to severe impairment of lung function with a potentially fatal outcome. ${ }^{57}$ More recent reports suggest that physiological abnormalities may occur in the absence of symptoms of dyspnoea, cough, fever, weight loss, or pleuritic chest pain. ${ }^{13}$

In an attempt to identify patients with adverse pulmonary reactions to amiodarone early in the course of their disease, other investigators have studied pulmonary function in symptom free patients. In a prospective study of 35 patients who received amiodarone for a mean of 19.3 months, Rakita et al ${ }^{14}$ 
found no relationship between cumulative drug dose and $F_{1} V_{1} / F V C$, TLC, or TLCo. Serial pulmonary function studies appear to be of no value for the prediction of pulmonary toxicity. Other investigators have shown small decreases in TLC and FVC over a period ranging from six to nine months in asymptomatic patients receiving 400 to $413 \mathrm{mg}$ of amiodarone daily. ${ }^{1315}$ Kudenchuk et al ${ }^{16}$ suggested that adverse pulmonary complications were more common among patients with abnormal TLC and TLCO and an abnormal chest radiograph before the start of treatment. Five of our patients had no clinical, radiological, or pulmonary function evidence of lung disease. We believe that this group is representative of the population of otherwise unaffected patients taking amiodarone, and we found no evidence of alveolitis in these patients.

We found that in patients with pneumonitis-that is, those with radiological infiltrates for which no other cause was apparent-total lung capacity was reduced by a mean of $19.6 \%$ and vital capacity by $19.9 \%$ at the time of presentation. Moreover, cumulative drug dose per kilogram of body weight and the duration of drug treatment were significantly correlated with the degree of lung restriction as reflected by TLC $(\mathrm{r}=-0.91$ and -0.96$)$ and FVC $(\mathrm{r}=$ -0.90 and -0.85 ). The severity of the lung restriction induced by amiodarone appears to be related to the cumulative drug dose and duration of treatment among patients who develop the adverse drug reaction.

The presence of intra-alveolar foamy macrophages with hypertrophy of type 2 pneumocytes and alveolar septal widening with or without evidence of fibrosis have been reported in specimens obtained by either transbronchial biopsy ${ }^{17}$ or open lung biopsy ${ }^{7-9}$ from patients developing amiodarone induced pneumonitis. Electron microscopic examination of open lung biopsy specimens showed numerous membrane bound cytoplasmic inclusions composed of granular osmophilic material or concentric layers of membranous material in alveolar macrophages, interstitial cells, endothelial cells, and type 2 pneumocytes. ${ }^{918}$ The present study shows that amiodarone bodies were present in all subjects regardless of clinical state. Moreover, we have found highly significant relationships between the number of amiodarone bodies in alveolar macrophage transections, average daily dose $(r=0.65)$ and serum amiodarone concentration $(r=0.72)$ in our subjects.

The pathogenesis of amiodarone induced pneumonitis remains unresolved. Schwartz et al reported an increase in percentage of polymorphonuclear cells in bronchoalveolar lavage fluid (mean $24.7 \%$, range 6-79\%) in eight patients. ${ }^{17}$ Akoun et al suggested a process of cell mediated hypersensitivity in one patient, who had positive reactions in skin and basophil degranulation tests, an increase in percentage of lymphocytes ( $31 \%$ lymphocytes), an inverted ratio of helper to suppressor $\mathrm{T}$ lymphocytes in bronchoalveolar lavage fluid, secretion of leucocyte inhibition factor, and a positive result in the lymphoblastic transformation test after exposure to amiodarone. ${ }^{19}$ Our previous work and that of Marchlinski et al $^{920}$ suggested that the accumulation of amiodarone in body tissue may affect lipid metabolism and turnover of lysosomal membranes, thus including pulmonary toxicity through a metabolic pathway. No patients in the present study had proportional differential cell counts in their lavage fluid suggestive of active alveolitis. Furthermore, amiodarone bodies were present in alveolar macrophages in all patients exposed to amiodarone. The presence of amiodarone bodies in lavaged alveolar macrophages therefore serves as an index of previous drug exposure rather than as a marker of the presence or severity of disease. We conclude that, while the severity of the restrictive lung disease in patients developing amiodarone toxicity is related to cumulative drug dose corrected for body weight and duration of drug treatment, the development of lung toxicity is to some extent an idiosyncratic process.

We are indebted to Dr M Waxman, Dr D Cameron, and Dr S Lenkei for referring patients and to Dr D Chamberlain for performing differential cell counts on the lavage fluid. We thank MT Berk for secretarial assistance.

\section{References}

1 Fogoros RN, Anderson KP, Winkle RA, Swerdlow CD, Mason JW. Amiodarone: clinical efficacy and toxicity in 96 patients with recurrent, drug-refractory arrhythmias. Circulation 1983;68:88-94.

2 Heger JJ, Prystowsky EN, Jackman WM, Naggarelli GV, Warfel KA, Rinkenberger RL, Zipes DP. Amiodarone: clinical efficacy and electrophysiology during long-term therapy for recurrent ventricular tachycardia or ventricular fibrillation. $N$ Engl J Med 1981;305: $539-45$.

3 McGovern B, Garan H, Kelly E, Ruskin J. Adverse reactions during treatment with amiodarone hydrochloride. Br Med J 1983;287:175-80.

4 Harris L, McKenna W, Rowland E, Krikler D. Side effects and possible contraindications of amiodarone use. Am Heart J 1983;106:916-23.

5 Sobol SM, Rakita L. Pneumonitis and pulmonary fibrosis associated with amiodarone treatment: a possible complication of a new antiarrhythmic drug. Circulation 1982;65:819-24.

6 Riley SA, Williams SE, Cooke NJ. Alveolitis after treatment with amiodarone. $\mathrm{Br}$ Med J 1982;284:161-2.

7 Darmanata JI, van Zandwijk N, Duren DR, et al. Amiodarone pneumonitis: three further cases with a review 
of published reports. Thorax 1984;39:57-64.

8 Pollak PT, Sami M. Acute necrotizing pneumonitis and hyperglycemia after amiodarone therapy: case report and review of amiodarone-associated pulmonary disease. Am J Med 1984;76:935-9.

9 Marchlinski FE, Gansler TS, Waxman HL, Josephson ME. Amiodarone pulmonary toxicity. Ann Intern Med 1982;97:839-45.

10 Crystal RG, Gadek JE, Ferrans VJ, Fulmer JD, Line BR, Hunninghake GW. Interstitial lung disease: current concepts of pathogenesis, staging and therapy. Am J Med 1981;70:542-68.

11 Braude AC, Chamberlain DW, Rebuck AS. Proportional analysis of respiratory cells obtained by bronchoalveolar lavage. Can Med Ass J 1982;126:1401-3.

12 Canada AT, Lesko LJ, Haffajee CI. Disposition of amiodarone in patients with tachyarrhythmias. Curr Ther Res 1981;30:968-74.

13 Freedman AP, Speilman SR, Greenspan AM, Horowitz LN. Pulmonary function surveillance in amiodarone therapy [abstract]. Am Rev Respir Dis 1984;129, April suppl: A52.

14 Rakita L, Sobol SM, Mostow N, Vrobel T. Amiodarone pulmonary toxicity. Am Heart $J$ 1983;106:906-16.

15 Manresa F, Cequier A, Escarrabill J, Perez Ayuso MJ, Sabater X, Gausi C. Amiodarone and lung function [letter]. Lancet 1983;ii:1367.

16 Kudenchuk PJ, Pierson DJ, Greene HL, Graham EL, Sears GN, Trobaugh GB. Prospective evaluation of amiodarone pulmonary toxicity. Chest $1984 ; 86: 541-8$.

17 Schwartz JRL, Levin DC, Mojarad M, Friday KJ, Jackman WM, Lazzara R. Amiodarone-induced fibrosing alveolitis, a new drug related lung disease: diagnostic value of bronchoalveolar lavage and transbronchial biopsy. Am Rev Respir Dis 1984;129, April suppl: A71.

18 Colgan T, Simon GT, Kay JM, Pugsley SO, Eydt J. Amiodarone pulmonary toxicity. Ultrastruct Pathol 1984;6: 199-207.

19 Akoun GM, Gauthier-Rahman S, Milleron BJ, Perrot JY, Mayaud CM. Amiodarone-induced hypersensitivity pneumonitis: evidence of an immunological cellmediated mechanism. Chest 1984;85:133-5.

20 Downar E, Shaikh N, Butany JW. Amiodarone-a potent phospholipase inhibitor [abstract]. J Am Coll Cardiol 1984;3, Feb suppl: 604. 